\title{
Diverse Aspects of Worry among Younger and Older Adults: A Multifaceted Approach
}

\author{
Katie L. Granier, Daniel L. Segal \\ Department of Psychology, University of Colorado Colorado Springs, Colorado Springs, USA \\ Email: kgranier@uccs.edu,dsegal@uccs.edu
}

How to cite this paper: Granier, K. L., \& Segal, D. L. (2021). Diverse Aspects of Worry among Younger and Older Adults: A Multifaceted Approach. Psychology, 12, 441-461.

https://doi.org/10.4236/psych.2021.123028

Received: February 6, 2021

Accepted: March 28, 2021

Published: March 31, 2021

Copyright $\odot 2021$ by author(s) and Scientific Research Publishing Inc. This work is licensed under the Creative Commons Attribution International License (CC BY 4.0).

http://creativecommons.org/licenses/by/4.0/

\begin{abstract}
The aims of this study were to examine age-differences regarding various worry components among younger and older adults and to characterize the relationships between worry domains and anxiety. A total of 311 younger adults (18 - 30 years) and 100 older adults (65+ years) completed seven worry and anxiety questionnaires, focusing on worry content, worry severity, and responses to worry. A series of $2 \times 2$ ANOVAs was computed to examine differences in worry and anxiety related to age and gender. Interaction effects indicated that gender differences among younger adults were significantly more pronounced than older adults with women reporting greater worry and anxiety than men. Main effects of age indicated that younger adults reported greater worry than older across all worry domains. Worry and anxiety measures were found to be highly correlated across all domains. Findings indicate that worry is fundamentally related to anxiety across age groups and that a variety of aspects of worry should be considered to understand the breadth of worry's influence on anxiety. Additionally, age and gender have unique relationships with worry and anxiety and interact to complicate individuals' symptom profiles. Thus, worry is a multifaceted construct that is crucial to understanding anxiety in general and warrants multifaceted assessment and intervention.
\end{abstract}

\section{Keywords}

Anxiety, Worry Severity, Worry Content, Worry Domains, Aging, Psychopathology, Mental Health, Geropsychology

\section{Introduction}

Most people fret or worry, especially in times of high stress and adversity. Borkovec et al. (1983) proposed a prominent definition of worry in their early ex- 
plorations of the construct, describing worry as a relatively uncontrollable chain of negative thoughts or images manifested in an effort to problem-solve an issue with an uncertain outcome. Although worry has been notably recognized as a key symptom of anxiety disorders, even representing a component of the cardinal criterion for diagnosis of Generalized Anxiety Disorder (GAD), worry has recently been acknowledged as a dimensional construct that serves adaptive purposes when moderate levels are maintained. Specifically, worry can serve as a problem-solving strategy to minimize anxiety and preoccupation with possible outcomes of future events. Numerous models of worry have been developed to explain why and how worry may function this way, some of which were developed more generally for anxiety and have been later expanded to the discussion of worry. The Intolerance of Uncertainty Model describes worry in the context of individuals' fear of ambiguity and the unknown, and this model has been well supported (Dugas et al., 1998). The Cognitive Avoidance Model of Worry postulates that worry acts as a method of reducing or muffling negative responses and arousal of anxiety (Borkovec et al., 2004). It proposes that worry acts to buffer individuals from the full effects of fear and anxiety responses, subsequently being negatively reinforced as a coping strategy. The Contrast Avoidance Model posits that individuals prone to worrying maintain a fear of shifts from neutral or positive emotional states to negative emotional states, instead inducing and maintaining a steady negative emotional state by worrying to avoid downward shifts (Newman \& Llera, 2011). Lastly, the Emotion Dysregulation Model suggests that those with anxiety symptoms struggle to understand and regulate emotional arousal, resulting in maladaptive anxiety and worry patterns (Mennin et al., 2005). Though each of these models presents a unique perspective on the nature and functioning of worry, each one emphasizes discomfort with negative states and individuals' attempts to avoid and/or mitigate their effects.

\subsection{Worry in Late Life}

Research on worry in older adults is generally sparse, although it has been on the rise in response to the shifting views of worry's role in anxiety. Worrying in late life appears to be a relatively infrequent occurrence (Wisocki et al., 1986; Wisocki, 1994) that decreases in frequency as aging occurs, a finding supported across studies and methodologies (Goncalves \& Byrne, 2013; Basevitz, 2003). Regardless of the decreased frequency of worry in late life, however, worry often exerts a negative impact on older adults. For example, even mild worry and anxiety tendencies can influence and predict cognitive decline (Pietrzak et al., 2012) and worry negatively impacts several domains of cognition above and beyond the influence of anxiety and depression (de Vito et al., 2017). Worry also negatively influences the trajectory of anxiety symptoms independent of diagnoses of an anxiety disorder (Spinhoven et al., 2017) and is associated with greater depressive symptoms, decreased quality of life (Golden et al., 2011), greater distress (Miloyan \& Pachana, 2016), and increased risk of cardiovascular 
health complications (Tully et al., 2013) among older adults. However, even with these and other notable findings, worry is often viewed as a singular construct, with research primarily focusing on overall worry severity and glazing over its other multidimensional aspects. In this article, worry is collapsed into the following four components of interest: nature/constructiveness, content, severity, and response behaviors.

\subsection{The Multifaceted Nature of Worry}

A recent distinction has been made between worry that is adaptive and serves a productive purpose (termed "constructive worry") and worry that becomes harmful and persists over time in a distressing manner (termed "unconstructive worry"; McNeill \& Dunlop, 2016). Theories such as the Cognitive Avoidance Model of Worry and the Intolerance of Uncertainty Model suggest that worry may be an adaptive tool used to reduce anxiety by mitigating uncertainty surrounding anticipated events. This notion has been supported through studies showing the beneficial nature of moderate worry in healthy "non-worriers" when compared to pathological worriers (Ottaviani et al., 2014). While healthy controls' worry was adaptive in nature, acting in response to perceptions of threat, worry that was automatic and uncontrollable lacked adaptive utility and ultimately became harmful. Similarly, one study has indicated that attentional abilities and processes can be negatively influenced by states of elevated worry, whereas less frequent worrisome thoughts were associated with improved attentional performance (Fox et al., 2015). Wu et al. (2019) also found that when comparing those of varying worry tendencies, moderate worry maximized engagement of limbic-prefrontal functional connectivity whereas severe worry negatively impacted emotional regulation abilities, supporting the theory that moderate levels of worry (as opposed to persistent worry tendencies) can have functional benefits.

\subsection{Worry Content}

Worry content pertains to the subject matter of worrying thoughts and behaviors. Contents of worry differ across age groups, meeting expectancies based on current stage of life and primary concerns (i.e., younger adults report more worry about finances, whereas older adults report worry regarding relationships and health/independence; Hunt et al., 2009; Lindesay et al., 2006; Wisocki, 1994). Diefenbach et al. (2001) found that that the content of older adults' worries may have significant correspondence with determinations of mood and anxiety disorders. Specifically, they reported differences in the patterns of worry content across participants with anxiety disorders, mood disorders, and comorbid anxiety and mood disorders, with worries about "aimless future" being highly associated with depression, "loss of control" associated with anxiety, and a mixed profile of worries associated with comorbid anxiety and depression. However, Borkovec et al. (1991) posit that differences in worry content depend 
largely on life context rather than self-reported severity of worry. Even so, by analyzing and comparing the contents of worry, researchers may achieve greater understandings of the continuum between normal and pathological worry.

\subsection{Worry Severity}

Worry severity refers to the extent to which worry impacts and interferes with one's life. Ruscio (2002) noted that distinctions between self-reported "high worriers" with and without GAD have been determined by the severity and frequency of their worry patterns. These findings indicate that worry severity and frequency may play focal roles in determining the pathology of anxiety and may serve as indicators of disorder course. However, worry frequency shifts across the lifespan, with findings by Basevitz (2003) indicating that the frequency of worry decreases in late life and is typically a comparatively infrequent occurrence in older populations, findings which are consistent across the literature and supported across cultures (Hunt et al., 2009). Proposed explanations for these decreases include an increase in learned coping abilities as individuals age (Borkovec, 1988), lowered detection of anxiety symptoms in older adults, and changes in expression of worry and anxiety behaviors. In addition, decreased responsibilities (such as those related to employment) and potential cohort effects are other potential influencers in this decline (Basevitz, 2003). Additionally, patterns of worry frequency also seem to change as older adults continue to age. Jeon et al. (2006) indicate that while worries decrease as individuals approach late adulthood, older adults may experience greater worries as they advance through late life, with those in the oldest-old group expressing greater worries than the young-old. Nilsson and colleagues (2019) reported similar findings, noting that when comparing older adults in their seventies and eighties, autonomic arousal and clinical anxiety decreased while worry increased, demonstrating that the expression of anxiety and prevalence of worry may change based on age among older adults.

\subsection{Response to Worry}

Understanding reactions to worry and management styles can offer valuable information on the nature of worrying and provide a more comprehensive perspective on its maintenance and pervasiveness. Gould et al. (2018) examined self-reported emotional responses to worry induction, indicating that responses to worry vary across age groups, with endorsed emotions spanning across diagnostic categories. Emotional responses to worry spanned from anxiety-typical such as tense and stressed to depression-typical such as sad and discouraged and impulsivity or anger-related as in impatient and agitated, indicating a wide range of potential emotional responses and psychological associations in individuals' reactions to worry. It follows then that behavioral responses to worry may also vary. Some responses may be characteristic of safety behaviors, or active behaviors that mitigate the negative impacts of worry, such as making plans ahead of 
time to avoid worrying about missed obligations or engaging in self-soothing activities to prevent excessive worrying. On the other hand, individuals may engage in avoidance behaviors that perpetuate or exacerbate worry, for example by procrastinating or simply doing nothing.

\subsection{The Current Study}

In this study, we aimed to define and explore the structure of worry in older adults using a variety of domains to paint a comprehensive picture of worry as both an adaptive function and as a symptom of psychopathology. Whereas worry itself has been examined in relation to anxiety in much of the literature, we deconstructed these diverse aspects of worry to evaluate their specific relationships to anxiety in late life. Notably, these specific facets of worry have never been examined together within a single study. Thus, the inclusion of all of these variables together, particularly in a cross-sectional design including both younger and older adults, represents an approach that is novel and potentially important.

Two primary hypotheses were offered. First, it was hypothesized that there would be age differences on measures of worry and anxiety. It was anticipated that scores on the Penn State Worry Questionnaire (PSWQ), Worry Domains Questionnaire Short Form (WDQ-SF), Brief Measure of Worry Severity (BMWS), and Geriatric Anxiety Scale (GAS) would be meaningfully lower among older adults than younger adults, as suggested by the literature indicating that worry and anxiety tend to decrease in late life. Similarly, it was hypothesized that the two groups would differ on ratings of worry constructiveness, types of worry responses engaged in, and primary areas of concern. Specifically, it was hypothesized that older adults would experience greater constructive worry and engage in greater adaptive behaviors due to the accumulation of experience and coping skills across the lifespan, as well as experience elevated worry about health and social engagement compared to younger adults as suggested by the previous literature and common life concerns identified in late adulthood (e.g., increased medical diagnoses, risk of social isolation). Second, it was hypothesized that there would be strong positive correlations between total WDQ-SF, PSWQ, Worry Scale, and BMWS scores with GAS total scores, as increased worry severity is associated with increased anxiety. It was also hypothesized that there would be strong relationships between anxiety and worry constructiveness, as it is suggested that maladaptive worry exacerbates anxiety symptoms, whereas adaptive worry functions to decrease anxiety. Due to the presumed functioning of safety behaviors to reduce anxiety and avoidance behaviors to maintain it, it was hypothesized that there would be corresponding relationships between GAS and Worry Behavior Inventory (WBI) scores. These relationships were hypothesized to be consistent across age groups, as though cross-sectional differences were expected within each domain, the relationships between the domains themselves were anticipated to be stable based on their core functions (e.g., worry was expected to be positively related to anxiety regardless of age group as worry is a 
symptom of anxiety and they are inherently related). Thus, the current study sought to further the current understanding of worry in late adulthood by examining how these new and understudied facets of worry (i.e., constructiveness, response behaviors) relate to other domains of worry and contribute to overall experiences of worry and anxiety among younger and older adults.

\section{Method}

\subsection{Participants}

Younger adults. This sample (ages ranged from 18 to 30 years) included 311 participants (254 women; $M$ age $=20.24$ years; $S D=2.61$ ) recruited from an undergraduate psychology department's extra credit system. The sample comprised of 207 White individuals with the remaining identifying themselves as Hispanic ( $n$ $=38)$, multiracial/multiethnic $(n=27)$, Black $(n=17)$, East Asian $(n=12)$, or other. 208 of the younger adults had completed some college, with the remaining having a high school diploma or GED $(n=60)$, associate's degree $(n=37)$ or higher $(n=6)$.

Older adults. This sample (ages ranged from 65 to 79 years) included 100 participants (62 women; $M$ age $=68.82$ years; $S D=3.32$ ) recruited from Amazon's Mechanical Turk. The sample comprised of 89 White individuals with the remaining identifying themselves as Black $(n=4)$, Hispanic $(n=2)$, East Asian $(n$ $=1)$, multiracial/multiethnic $(n=1)$, or other. 20 older adults had completed some college, with the remaining having a high school diploma or GED $(n=10)$, associate's degree $(n=19)$, Bachelor's degree $(n=29)$, or higher $(n=22)$. Regarding socioeconomic status (SES), the samples were fairly equivalent in SES composition, with most participants in both groups identifying as working or middle class (see Table 1).

\subsection{Measures}

\subsubsection{The Worry Scale}

The Worry Scale is a 35-item self-report measure of worry content specifically created for use with older adults (Wisocki et al., 1986). Items provide assessment of concerns specific to older adults, addressing the lack of applicability found in other, similar measures. The Worry Scale has three sub-areas of worry content: finances (5 items), health (17 items), and social conditions (13 items). Participants rate how much they worry about each item on a 5-point Likert-type scale. Responses are scored by adding the total values of each item's rating to get a cumulative score as well as content area scores. The Worry Scale demonstrated excellent internal consistency across both groups in the current study (younger: $\alpha=0.96$; older: $\alpha=0.97$ ).

\subsubsection{Geriatric Anxiety Scale (GAS)}

The GAS is a self-report measure designed to assess anxiety among older adults (Segal et al., 2010). Respondents are instructed to rate how often each item 
Table 1. Demographic characteristics of study sample.

\begin{tabular}{|c|c|c|}
\hline & Younger adults & Older adults \\
\hline \multicolumn{3}{|l|}{ Gender } \\
\hline$N$ & 311 & 100 \\
\hline$\%$ Female & 81.70 & 62 \\
\hline$\%$ Male & 16.40 & 38 \\
\hline$\%$ Transgender & 0.60 & 0 \\
\hline$\%$ Nonbinary/Androgynous & 1 & 0 \\
\hline \multicolumn{3}{|l|}{ Race } \\
\hline$N$ & 311 & 100 \\
\hline$\%$ White & 66.60 & 89 \\
\hline$\%$ Black & 5.50 & 4 \\
\hline$\%$ American Indian/Alaskan Native & 0.30 & 0 \\
\hline$\%$ East Asian & 3.90 & 1 \\
\hline$\%$ West Asian & 0.30 & 0 \\
\hline$\%$ Native Hawaiian/Pacific Islander & 0.30 & 0 \\
\hline$\%$ Hispanic/Latinx & 12.20 & 2 \\
\hline$\%$ Multiracial/multiethnic & 8.70 & 1 \\
\hline$\%$ Other & 0.30 & 2 \\
\hline \multicolumn{3}{|l|}{ Education } \\
\hline$N$ & 311 & 100 \\
\hline \% High school diploma/GED & 19.30 & 10 \\
\hline$\%$ Some College & 66.90 & 20 \\
\hline$\%$ Associates degree & 11.90 & 19 \\
\hline$\%$ Bachelors degree & 1.60 & 29 \\
\hline \% Some graduate school & 0.30 & 4 \\
\hline$\%$ Masters degree & 0 & 15 \\
\hline \% Doctoral degree & 0 & 3 \\
\hline \multicolumn{3}{|l|}{ SES } \\
\hline$N$ & 311 & 100 \\
\hline$\%$ Affluent/wealthy & 1 & 0 \\
\hline$\%$ Upper middle class & 12.90 & 11 \\
\hline$\%$ Middle Class & 44.10 & 49 \\
\hline$\%$ Working Class & 25.40 & 21 \\
\hline \% Working Poor & 7.40 & 12 \\
\hline$\%$ Poverty Level & 1.90 & 7 \\
\hline
\end{tabular}

Note. Missing percentages for the race and SES variables are indicative of participant responses of "prefer not to answer/do not know". SES = Socioeconomic status. 
occurs on a 4-point Likert-type scale. Respondents' ratings are summed for a total score, with higher composite scores indicating higher levels of anxiety. The GAS has 25 scored items measuring three domains (somatic, affective, and cognitive symptoms). It also includes five non-scored items pertaining to specific areas of concern for respondents. Although designed for older adults, the GAS is appropriate for use across adulthood (Segal et al., 2010) and has strong evidence for validity for use with diverse samples of older adults (Segal, Granier, Stone, \& Pifer, 2019). The GAS total score showed excellent reliability among both groups in the current study (younger: $\alpha=0.95$; older: $\alpha=0.95$ ). The internal consistency of the GAS subscale scores ranged from good to excellent: somatic (younger: $\alpha=$ 0.85 ; older: $\alpha=0.82$ ), affective (younger: $\alpha=0.89$; older: $\alpha=0.87$ ), and cognitive (younger: $\alpha=0.91$; older: $\alpha=0.87$ ).

\subsubsection{Worry Domains Questionnaire Short Form (WDQ-SF)}

The WDQ is a self-report questionnaire designed to evaluate non-pathological worry (Tallis et al., 1992). The WDQ assesses worry across five content areas: relationships, aimless future, work, financial, and lack of confidence. The WDQ-SF is a shortened version of the original WDQ intended for quicker administration and simplicity (Stöber \& Joorman, 2001). It includes 10 items measured on a 5-point Likert-type scale from 0-not at all to 5-extremely. This version was used in the current study due to higher applicability for older adults due to fewer items assessing the "work" domain. In addition, the measure was slightly modified. Two items were altered to increase relevance for older adult participants. Item number seven "that I will not keep my workload up to date" was modified to instead state "that I will not keep my work or hobbies up to date" and item number eight "that I leave work unfinished" was modified to state "that I leave work or projects unfinished." The WDQ-SF showed excellent reliability in the current study (younger: $\alpha=0.91$; older: $\alpha=0.90$ ).

\subsubsection{Penn State Worry Questionnaire (PSWQ)}

The PSWQ is a 16-item self-report measure widely used to measure worry severity (Meyer et al., 1990). Respondents are instructed to rate how typical each item is of them on a 5-point Likert-type scale from 0-not at all typical of me to 5-very typical of me. A total score is obtained by summing responses and reverse-scoring five items, with a higher total score indicating greater worry severity. The PSWQ demonstrated excellent reliability across both age groups in the current study (younger: $\alpha=0.93$; older: $\alpha=0.97$ ).

\subsubsection{Worry Behaviors Inventory (WBI)}

The WBI is a relatively new 10 -item self-report measure assessing behavioral responses to worry (Mahoney et al., 2016). Respondents are instructed to rate how often they do each of the 10 behaviors on a 5-point Likert-type scale ranging from 0 -none of the time to 4-all of the time. Items are divided into three avoidance behaviors and seven safety behaviors. An avoidance behavior total score can be obtained by summing ratings from the three avoidance items for a 
maximum score of 12 , and a safety behaviors score may be obtained by summing the remaining seven items for a maximum score of 28 . The WBI has been validated for use with diverse adult populations, though specific data for use with older adults has not yet been established (Mahoney et al., 2016; Mahoney et al., 2018). The WBI avoidance scale showed acceptable reliability among both groups in the current study (younger: $\alpha=0.72$; older: $\alpha=0.70$ ) and the safety scale showed good reliability among both groups (younger: $\alpha=0.85$; older: $\alpha=0.86$ ).

\subsubsection{Brief Measure of Worry Severity (BMWS)}

The BMWS is an 8-item self-report measure of the severity of worry (Gladstone et al., 2005). The BMWS comprises a 4-point Likert-type scale with responses ranging from 0-not true at all to 3-definitely true. Responses are summed to get a total score, with higher composite scores indicating higher levels of severity. The BMWS has been shown to have good validity, making it a useful tool in assessing the severity of worry. Though older adult participants were included in the original validation sample, no previous studies have utilized the BMWS in an older adult sample independent of other age groups. The BMWS displayed excellent reliability across both groups in the current study (younger: $\alpha=0.92$; older: $\alpha=0.94$ ).

\subsubsection{Constructive and Unconstructive Worry Questionnaire (CUWQ)}

The CUWQ is a recently developed tool created to assess differences between adaptive and maladaptive worry (McNeill \& Dunlop, 2016). The scale consists of 18 self-report items and respondents are instructed to report on a 7-point Likert-type scale from 1-does not apply to me, when I worry to 7-applies very strongly to me, when I worry. Scores may be obtained by summing items by prespecified domains or by summing item ratings by hypothesized nature (constructive vs. unconstructive). Both subscales demonstrated convergent validity with other worry measures (i.e., the PSWQ and the WDQ-SF), and demonstrated good to excellent internal consistency (McNeill \& Dunlop, 2016). The CUWQ has not been used with an exclusively older adult sample in previous research. In the current study, both the unconstructive scale (younger: $\alpha=0.91$; older: $\alpha=$ 0.95 ) and the constructive scale (younger: $\alpha=0.88$; older: $\alpha=0.93$ ) of the CUWQ demonstrated good to excellent reliability.

\subsection{Procedure}

Following approval from the Institutional Review Board (IRB), all measures and informed consent were uploaded to Qualtrics for data collection. Participants completed the electronic consent process and responded to each self-report measure before receiving a debriefing statement and confirmation of completion. The Qualtrics survey was linked to an Amazon MTurk study through which older adult participants received compensation of $\$ 1.25$ for their participation. MTurk participant selection features were utilized to limit visibility of the study to only older adult users. The survey was advertised until the limit of 
105 participants was reached. Younger adult participants were recruited via undergraduate courses on a university campus within the U.S. They were provided a link to the Qualtrics survey via the psychology department research system, and they received extra credit in psychology courses for their participation. Following data collection, all survey responses were compiled and analyzed using SPSS (v25) statistical software.

\section{Results}

Little's MCAR was performed on the original dataset to determine the pattern of missing data among the quantitative data variables. Little's MCAR test was nonsignificant, Chi-Square $=8417.44, d f=8710, p=0.99$, indicating that the missing data were missing at random. Multiple imputation was used to impute missing data for all quantitative variables except for demographic responses. A total of 50 imputations were run to account for missing data. When available, pooled results are reported. Prior to imputation, $0.16 \%$ of all quantitative variable values were missing, representing very little missing data.

\subsection{Cross-Sectional (Age-Differences) Results}

Prior to completing the cross-sectional analyses, chi-square tests were completed to determine whether the younger and older adult groups differed based on gender. Chi-square analyses indicated that younger adults (81.9\%) were significantly more likely to be female (versus male) than older adults $(62 \%), \mathrm{x}^{2}(2)=$ $21.69, p<0.001$. Due to the significant gender differences between the groups, gender was statistically accounted for throughout subsequent analyses. Additionally, simple correlations were computed to examine the influence of race on the outcome variables. Among the younger adult sample, race was found to be unrelated to all measures of worry and anxiety. Among the older adult sample, identifying as White was associated with less engagement in both safety and avoidance behaviors compared to ethnic minorities $(r=0.24, p<0.05 ; r=0.38$, $p<0.001$, respectively).

A series of $2 \times 2$ independent samples ANOVAs were computed to examine age group and gender differences on worry and anxiety severity as measured by the PSWQ, BMWS, WDQ-SF, and GAS. ANOVA results and effect sizes (reported as Cohen's $d$ ) are based on the original, non-imputed dataset. Among all worry and anxiety severity measures (i.e., PSWQ, BMWS, WDQ-SF, and GAS), significant interaction effects were found between age and gender (see Figure 1). There was a significant interaction effect between gender and age group on worry severity as measured by the PSWQ $(F[1,393]=4.28, p<0.05)$, WDQ-SF $(F[1,397]=8.42, p<0.01)$, and BMWS $(F[1,396]=10.41, p<0.01)$ in that gender had a larger impact on worry severity among younger adults than older adults. There was also a significant interaction effect between gender and age group on anxiety severity (GAS total) in that women reported greater levels of anxiety than men among the younger adult group, but the older adult group reported consistent anxiety across genders, $F(1,384)=9.78, p<0.01$. 

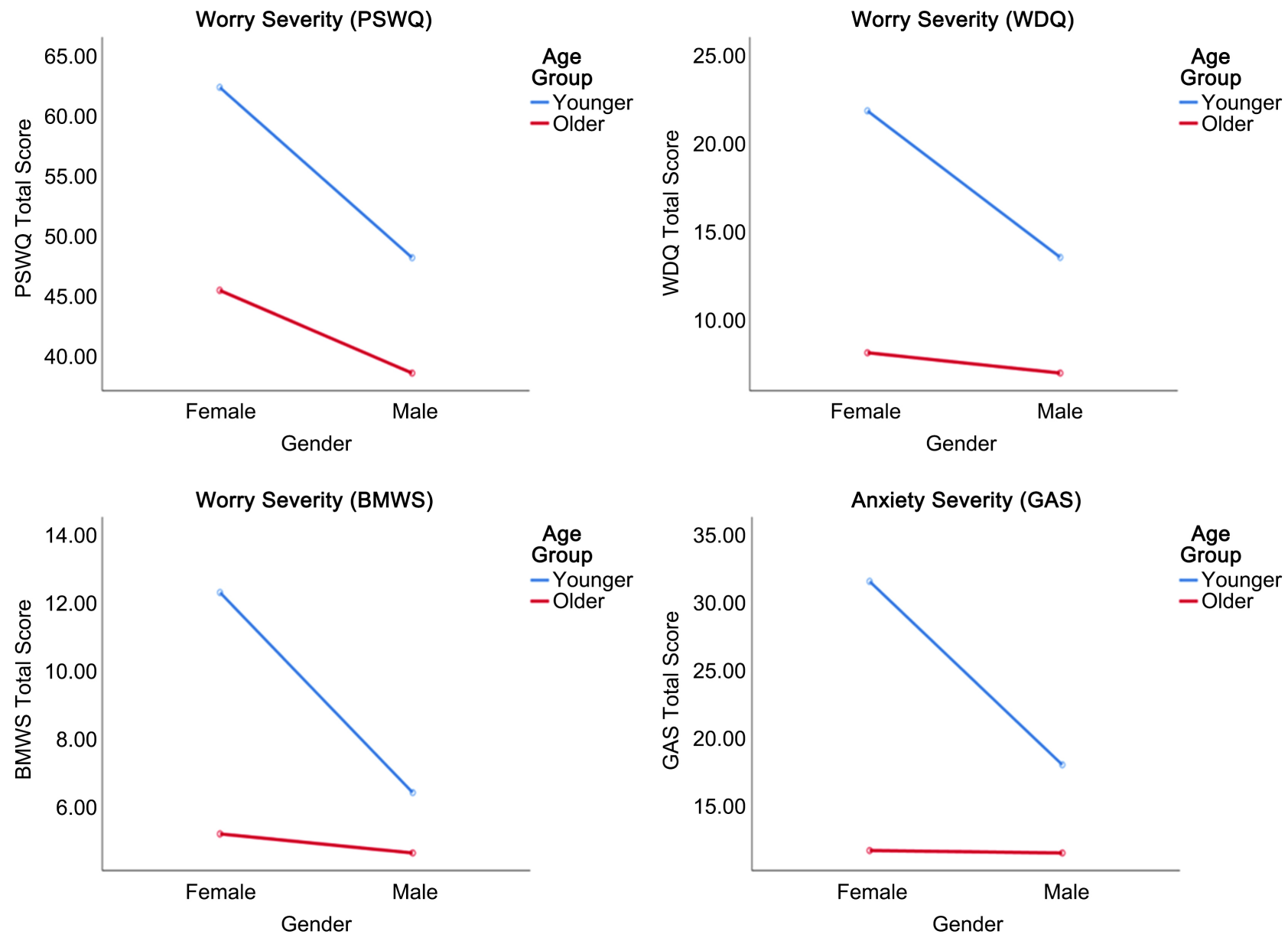

Figure 1. Interaction effects of age and gender on measures of worry and anxiety severity.

Additional $2 \times 2$ ANOVAs were computed to examine age and gender differences on worry content and responses as measured by the WDQ-SF, Worry Scale, and WBI. Significant interaction effects between age and gender were found on four subscales of the WDQ-SF: work $(F[1,401]=4.12, p<0.05)$, aimless future $(F[1,397]=4.39, p<0.05)$, low confidence $(F[1,401]=12.89, p<$ $0.001)$, and relationships $(F[1,401]=11.72, p<0.01)$ and the social subscale of the Worry Scale $(F[1,391]=6.62, p<0.05)$ with similar patterns to those found among worry and anxiety severity. There was also an interaction effect on the avoidance scale of the WBI, $F(1,396)=6.41, p<0.05$. As with worry severity, both younger and older women reported greater worry on these domains than their male counterparts, however gender differences were more distinct among younger adults. $2 \times 2$ ANOVAs were then run to examine age and gender's influence on the constructiveness of worry, however there were no interaction effects on either measure of worry constructiveness. There was also no main effect of age $(F[1,398]=0.57, d=0.10, p=0.45)$ on constructive worry. However, there was a main effect of age on unconstructive worry, $F(1,398)=37.22, d=$ $0.98, p<0.001$, with a large effect size such that younger adults reported greater unconstructive worry than older adults. 
A series of one-way between-subjects ANOVAs was then computed to examine the simple main effects of age among both male and female participants (See Table 2). Among women, there was a simple main effect of age on worry and anxiety severity as measured by the PSWQ $(F[1,307]=79.40, d=1.12, p<$ $0.001)$, WDQ-SF $(F[1,311]=96.78, d=1.55, p<0.001)$, $\operatorname{BMWS}(F[1,311]=$ $60.89, d=1.17, p<0.001)$, and GAS $(F[1,299]=62.88, d=1.73, p<0.001)$ such that younger women reported significantly greater worry and anxiety than older women, all with large effect sizes. Among men, there was a simple main effect of age on worry severity as measured by the PSWQ $(F[1,86]=8.77, d=0.63, p<$ $0.01)$ and WDQ-SF $(F(1,86)=12.03, d=0.75, p<0.01)$ with medium effect sizes such that younger men reported greater worry than older men, but no main effects were evident among BMWS or GAS scores. Thus, younger adults consistently reported greater worry and anxiety symptoms across genders, though findings were stronger among women than men.

Table 2. Simple main effects of age on measures of worry and anxiety.

\begin{tabular}{|c|c|c|c|c|c|c|c|c|c|c|c|c|}
\hline \multicolumn{13}{|c|}{ Age Simple Main Effects } \\
\hline \multirow{3}{*}{ Measure } & \multicolumn{6}{|c|}{ Female } & \multicolumn{6}{|c|}{ Male } \\
\hline & \multicolumn{2}{|c|}{ Younger } & \multicolumn{2}{|c|}{ Older } & \multirow{2}{*}{$F$} & \multirow{2}{*}{$d$} & \multicolumn{2}{|c|}{ Younger } & \multicolumn{2}{|c|}{ Older } & \multirow{2}{*}{$F$} & \multirow{2}{*}{$d$} \\
\hline & $M$ & $S D$ & $M$ & $S D$ & & & $M$ & $S D$ & $M$ & $S D$ & & \\
\hline GAS & 31.54 & 17.14 & 11.67 & 11.25 & $62.88^{\star * *}$ & $1.73^{3}$ & 18.00 & 15.84 & 11.49 & 14.26 & 3.91 & $0.43^{1}$ \\
\hline PSWQ & 62.27 & 11.75 & 45.36 & 17.84 & $79.40^{\star * *}$ & $1.12^{3}$ & 48.08 & 13.81 & 38.46 & 16.61 & $8.77^{\star *}$ & $0.63^{2}$ \\
\hline BMWS & 12.29 & 6.53 & 5.17 & 5.56 & $60.89^{* * *}$ & $1.17^{3}$ & 6.39 & 6.43 & 4.61 & 6.49 & 1.63 & $0.28^{1}$ \\
\hline WDQ-SF Total & 21.80 & 10.23 & 8.05 & 7.26 & $96.78^{\star * *}$ & $1.55^{3}$ & 13.48 & 9.14 & 6.89 & 8.39 & $12.03^{* *}$ & $0.75^{2}$ \\
\hline Avoidance & 5.63 & 2.99 & 3.08 & 2.53 & $37.64^{\star * *}$ & $0.92^{3}$ & 3.90 & 2.89 & 3.08 & 2.53 & 2.53 & $0.30^{1}$ \\
\hline Safety & 13.74 & 6.62 & 8.85 & 5.48 & $28.89^{\star \star *}$ & $0.80^{3}$ & 10.84 & 7.25 & 8.85 & 5.48 & 2.72 & $0.31^{1}$ \\
\hline WDQ-SF Work & 3.50 & 2.52 & 1.13 & 1.68 & $49.79^{* * *}$ & $1.11^{3}$ & 2.57 & 2.56 & 1.13 & 1.68 & $12.84^{* *}$ & $0.67^{2}$ \\
\hline WDQ-SF Finances & 4.39 & 2.73 & 3.11 & 2.61 & $10.82^{* *}$ & $0.48^{1}$ & 2.90 & 2.70 & 3.11 & 2.61 & .18 & 0.08 \\
\hline WDQ-SF Aimless Future & 4.98 & 2.59 & 1.30 & 1.99 & $105.77^{\star * *}$ & $1.59^{3}$ & 3.76 & 2.62 & 1.30 & 1.99 & $31.29^{* * *}$ & $1.06^{3}$ \\
\hline WDQ-SF Low Confidence & 4.74 & 2.54 & 1.32 & 1.82 & $99.57^{\star * *}$ & $1.55^{3}$ & 2.31 & 2.41 & 1.32 & 1.82 & $6.20^{*}$ & $0.46^{1}$ \\
\hline WDQ-SF Relationships & 4.16 & 2.43 & 1.16 & 1.23 & $88.76^{* * *}$ & $1.56^{3}$ & 2.22 & 2.27 & 1.16 & 1.23 & $9.89^{* *}$ & $0.58^{2}$ \\
\hline WS Finances & 7.60 & 4.50 & 5.60 & 4.78 & $9.65^{* *}$ & $0.43^{1}$ & 4.80 & 4.16 & 5.60 & 4.78 & .86 & 0.18 \\
\hline WS Health & 15.68 & 13.84 & 16.92 & 13.64 & .39 & 0.09 & 9.27 & 9.92 & 16.92 & 13.64 & $10.64^{\star \star}$ & $0.64^{2}$ \\
\hline WS Social & 18.08 & 13.22 & 10.64 & 10.18 & $16.39^{* * *}$ & $0.63^{2}$ & 8.26 & 9.97 & 10.64 & 10.18 & 1.47 & $0.24^{1}$ \\
\hline
\end{tabular}

Note. Significance attained at the ${ }^{\star} p<0.05 ;{ }^{* *} p<0.01 ;{ }^{* *} p<0.001$ level (2-tailed). ${ }^{1}$ indicates a small effect size. ${ }^{2}$ indicates a medium effect size. ${ }^{3}$ indicates a large effect size. GAS = Geriatric Anxiety Scale. PSWQ = Penn State Worry Questionnaire. BMWS = Brief Measure of Worry Severity. WDQ-SF $=$ Worry Domains Questionnaire Short Form. Avoidance = avoidance scale of the WBI. Safety = safety scale of the WBI. WS $=$ Worry Scale. Results are reported from a series of one-way between-subjects ANOVAs. 
Results of one-way ANOVAs comparing scores on worry content indicate that among women, younger adults endorsed significantly greater worry than older adults about work $(F[1,314]=49.74, d=1.11, p<0.001)$, finances $(F[1,313]=$ $10.82, d=0.48, p<0.01)$, aimless future $(F[1,311]=105.77, d=1.59, p<0.001)$, low confidence $(F[1,314]=99.57, d=1.55, p<0.001)$, and relationships $(F[1,314]$ $=88.76, d=1.56, p<0.001)$ on the WDQ-SF. Younger women also endorsed greater worry about social concerns $(F[1,308]=16.39, d=0.63, p<0.001)$ and finances $(F[1,314]=9.65, d=0.43, p<0.01)$ on the Worry Scale, but did not endorse significantly different amounts of worry about health compared to older women. Men demonstrated similar patterns of worry endorsement with younger adults endorsing significantly greater worry than older adults about work $(F[1$, $111]=12.84, d=0.67, p<0.01)$, aimless future $(F[1,108]=31.29, d=1.06, p<$ $0.001)$, low confidence $(F[1,111]=6.20, d=0.46, p<0.05)$, and relationships $(F[1,111]=9.89, d=0.58, p<0.01)$ on the WDQ-SF. However, on the Worry Scale, older men endorsed significantly greater worry about health $(F[1,107]=$ $10.64, d=0.64, p<0.01)$. Younger and older men did not endorse significantly different amounts of worry on the other worry content areas. Thus, younger adults tend to experience greater worry across a variety of worry content areas, though this difference is most prevalent among women, with both younger and older men experiencing less worry in general. However, these differences do not extend to the area of health concerns wherein women report similar amounts of worry and older men experience much greater worry then younger men. Overall, older adults endorsed the greatest amount of worry about health $(M=16.69)$ whereas younger adults endorsed the most worry about finances $(M=7.11)$ and social concerns $(M=16.59)$ on the Worry Scale.

In terms of worry responses, younger women endorsed using greater safety $(F[1,314]=28.89, d=0.80, p<0.001)$ and avoidance behaviors $(F[1,310]=$ $37.64, d=0.92, p<0.001)$ in response to worry compared to older women on the WBI. Among men, however, there were no differences between worry responses of younger and older adults. Thus, hypothesis one was partially supported by the current findings in that younger adults consistently reported greater worry severity, anxiety, and unconstructive worry. Also consistent with the hypothesis, age differences were found among avoidance behaviors and worry content areas, though these findings differed based on gender. Contrary to the hypothesis, younger adults also reported greater constructive worry and safety behaviors (among women) compared to older adults.

\subsection{Correlational Results}

A series of simple correlations were computed among the major variables in this study separately by age group (See Table 3 and Table 4). As hypothesized, all measures of overall worry and anxiety were significantly positively correlated. Specifically, the GAS, BMWS, PSWQ, Worry Scale, and WDQ-SF total scores were all significantly positively correlated at the $p<0.001$ level among both the younger adult sample and the older adult sample, with large effect sizes. 
Table 3. Correlations of major study variables among the younger adult sample.

\begin{tabular}{|c|c|c|c|c|c|c|c|c|c|c|c|c|c|c|}
\hline & 1 & 2 & 3 & 4 & 5 & 6 & 7 & 8 & 9 & 10 & 11 & 12 & 13 & 14 \\
\hline 1) Safety Scale & 1 & $0.65^{*}$ & $0.57^{*}$ & $0.49^{*}$ & $0.45^{*}$ & $0.60^{*}$ & $0.50^{*}$ & $0.50^{*}$ & $0.52^{*}$ & $0.57^{*}$ & $0.60^{*}$ & $0.53^{*}$ & -0.16 & 0.01 \\
\hline 2) Avoidance Scale & $0.65^{*}$ & 1 & $0.50^{*}$ & $0.50^{*}$ & $0.23^{*}$ & $0.55^{*}$ & $0.44^{*}$ & $0.39^{*}$ & $0.47^{*}$ & $0.53^{*}$ & $0.61^{*}$ & $0.53^{*}$ & $-0.20^{*}$ & 0.07 \\
\hline 3) GAS Total & $0.57^{\star}$ & $0.50^{*}$ & 1 & $0.67^{*}$ & 0.18 & $0.72^{*}$ & $0.72^{*}$ & $0.89^{*}$ & $0.94^{*}$ & $0.94^{*}$ & $0.76^{*}$ & $0.68^{*}$ & $-0.29^{*}$ & -0.02 \\
\hline 4) PSWQ & $0.49^{*}$ & $0.50^{*}$ & $0.67^{\star}$ & 1 & 0.15 & $0.73^{*}$ & $0.52^{*}$ & $0.52^{*}$ & $0.67^{\star}$ & $0.65^{*}$ & $0.74^{*}$ & $0.61^{*}$ & $-0.40^{*}$ & -0.04 \\
\hline 5) Constructive & $0.45^{*}$ & $0.23^{*}$ & 0.18 & 0.15 & 1 & $0.32^{*}$ & $0.20^{*}$ & 0.18 & 0.14 & 0.19 & 0.17 & 0.12 & -0.05 & -0.03 \\
\hline 6) Unconstructive & $0.60^{*}$ & $0.55^{*}$ & $0.72^{*}$ & $0.73^{*}$ & $0.32^{*}$ & 1 & $0.62^{*}$ & $0.58^{*}$ & $0.72^{*}$ & $0.69^{*}$ & $0.82^{*}$ & $0.67^{*}$ & $-0.31^{*}$ & -0.02 \\
\hline 7) Worry Scale & $0.50^{*}$ & $0.44^{*}$ & $0.72^{*}$ & $0.52^{*}$ & $0.20^{*}$ & $0.62^{*}$ & 1 & $0.60^{*}$ & $0.70^{*}$ & $0.68^{*}$ & $0.61^{*}$ & $0.67^{*}$ & $-0.25^{*}$ & -0.04 \\
\hline 8) GAS Somatic & $0.50^{*}$ & $0.39^{*}$ & $0.89^{*}$ & $0.52^{*}$ & 0.18 & $0.58^{*}$ & $0.60^{*}$ & 1 & $0.73^{*}$ & $0.75^{*}$ & $0.64^{*}$ & $0.52^{*}$ & $-0.26^{*}$ & -0.03 \\
\hline 9) GAS Cognitive & $0.52^{*}$ & $0.47^{*}$ & $0.94^{*}$ & $0.67^{*}$ & 0.14 & $0.72^{\star}$ & $0.70^{*}$ & $0.73^{*}$ & 1 & $0.86^{*}$ & $0.73^{*}$ & $0.66^{*}$ & $-0.28^{\star}$ & -0.04 \\
\hline 10) GAS Affective & $0.57^{\star}$ & $0.53^{*}$ & $0.94^{*}$ & $0.65^{*}$ & 0.19 & $0.69^{*}$ & $0.68^{*}$ & $0.75^{*}$ & $0.86^{*}$ & 1 & $0.73^{*}$ & $0.70^{*}$ & $-0.25^{*}$ & 0.01 \\
\hline 11) BMWS & $0.60^{*}$ & $0.61^{*}$ & $0.76^{*}$ & $0.74^{*}$ & 0.17 & $0.82^{*}$ & $0.61^{*}$ & $0.64^{*}$ & $0.73^{*}$ & $0.73^{*}$ & 1 & $0.69^{*}$ & $-0.32^{*}$ & 0.01 \\
\hline 12) WDQ-SF & $0.53^{*}$ & $0.53^{*}$ & $0.68^{*}$ & $0.61^{*}$ & 0.12 & $0.67^{*}$ & $0.67^{*}$ & $0.52^{*}$ & $0.66^{*}$ & $0.70^{*}$ & $0.69^{*}$ & 1 & $-0.28^{*}$ & -0.02 \\
\hline 13) Gender & -0.16 & $-0.20^{*}$ & $-0.29^{*}$ & $-0.40^{*}$ & -0.05 & $-0.31^{*}$ & $-0.25^{*}$ & $-0.26^{*}$ & $-0.28^{*}$ & $-0.25^{*}$ & $-0.32^{*}$ & $-0.28^{*}$ & 1 & 0.04 \\
\hline 14) Race & 0.01 & 0.07 & -0.02 & -0.04 & -0.03 & -0.02 & -0.04 & -0.03 & -0.04 & 0.01 & 0.01 & -0.02 & 0.04 & 1 \\
\hline
\end{tabular}

Note. ${ }^{*}$ Significance attained at the $p<0.001$ level (2-tailed). Bold text indicates $p<0.01$. Italic font indicates $p<0.05$. Constructive and Unconstructive refer to the subscales of the Constructive and Unconstructive Worry Questionnaire. Safety and Avoidance Scales refer to the subscales of the Worry Behaviors Inventory. PSWQ = Penn State Worry Questionnaire. GAS = Geriatric Anxiety Scale. BMWS = Brief Measure of Worry Severity. WDQ-SF $=$ Worry Domains Questionnaire Short Form. All results are pooled. Race was recoded as a binary variable (White and non-White) for correlational results due to small group sizes.

Table 4. Correlations of major study variables among the older adult sample.

\begin{tabular}{|c|c|c|c|c|c|c|c|c|c|c|c|c|c|c|}
\hline & 1 & 2 & 3 & 4 & 5 & 6 & 7 & 8 & 9 & 10 & 11 & 12 & 13 & 14 \\
\hline 1) Safety Scale & 1 & $0.54^{\star}$ & $0.57^{\star}$ & $0.58^{\star}$ & $0.44^{\star}$ & $0.52^{*}$ & $0.55^{\star}$ & $0.49^{*}$ & $0.58^{\star}$ & $0.53^{\star}$ & $0.45^{\star}$ & $0.46^{\star}$ & -0.04 & 0.24 \\
\hline 2) Avoidance Scale & $0.54^{\star}$ & 1 & $0.59^{*}$ & $0.50^{*}$ & 0.21 & $0.53^{*}$ & $0.51^{\star}$ & $0.56^{*}$ & $0.58^{\star}$ & $0.52^{*}$ & $0.55^{*}$ & $0.54^{\star}$ & 0.03 & $0.38^{\star}$ \\
\hline 3) GAS Total & $0.57^{\star}$ & $0.59^{*}$ & 1 & $0.74^{*}$ & 0.24 & $0.78^{*}$ & $0.79^{*}$ & $0.92^{*}$ & $0.96^{*}$ & $0.93^{*}$ & $0.86^{*}$ & $0.71^{*}$ & 0.01 & 0.12 \\
\hline 4) PSWQ & $0.58^{\star}$ & $0.50^{*}$ & $0.74^{*}$ & 1 & 0.22 & $0.78^{*}$ & $0.73^{*}$ & $0.67^{\star}$ & $0.73^{*}$ & $0.68^{*}$ & $0.73^{*}$ & $0.64^{*}$ & -0.18 & 0.12 \\
\hline 5) Constructive & $0.44^{\star}$ & 0.21 & 0.24 & 0.22 & 1 & $0.42^{\star}$ & 0.26 & 0.22 & 0.22 & 0.25 & 0.21 & $0.37^{\star}$ & -0.10 & 0.10 \\
\hline 6) Unconstructive & $0.52^{\star}$ & $0.53^{\star}$ & $0.78^{*}$ & $0.78^{\star}$ & $0.42^{\star}$ & 1 & $0.74^{\star}$ & $0.68^{*}$ & $0.76^{\star}$ & $0.76^{*}$ & $0.79^{*}$ & $0.78^{\star}$ & -0.19 & 0.04 \\
\hline 7) Worry Scale & $0.55^{\star}$ & $0.51^{\star}$ & $0.79^{*}$ & $0.73^{\star}$ & $0.26^{*}$ & $0.74^{*}$ & 1 & $0.75^{*}$ & $0.75^{\star}$ & $0.73^{*}$ & $0.67^{\star}$ & $0.67^{*}$ & -0.08 & 0.08 \\
\hline 8) GAS Somatic & $0.49^{\star}$ & $0.56^{*}$ & $0.92^{*}$ & $0.67^{\star}$ & 0.22 & $0.68^{*}$ & $0.75^{\star}$ & 1 & $0.81^{\star}$ & $0.75^{\star}$ & $0.75^{\star}$ & $0.56^{*}$ & -0.02 & 0.11 \\
\hline 9) GAS Cognitive & $0.58^{*}$ & $0.58^{*}$ & $0.96^{*}$ & $0.73^{*}$ & 0.22 & $0.76^{*}$ & $0.75^{\star}$ & $0.81^{\star}$ & 1 & $0.89^{*}$ & $0.83^{*}$ & $0.71^{\star}$ & 0.02 & 0.11 \\
\hline 11) BMWS & $0.45^{\star}$ & $0.55^{\star}$ & $0.86^{*}$ & $0.73^{\star}$ & 0.21 & $0.79^{*}$ & $0.67^{\star}$ & $0.75^{\star}$ & $0.83^{*}$ & $0.83^{*}$ & 1 & $0.73^{*}$ & -0.03 & 0.13 \\
\hline 12) WDQ-SF & $0.46^{*}$ & $0.54^{\star}$ & $0.71^{*}$ & $0.64^{\star}$ & $0.37^{\star}$ & $0.78^{*}$ & $0.67^{\star}$ & $0.56^{*}$ & $0.71^{\star}$ & $0.73^{*}$ & $0.73^{\star}$ & 1 & -0.07 & 0.06 \\
\hline 13) Gender & -0.04 & 0.03 & 0.01 & -0.18 & -0.10 & -0.19 & -0.08 & -0.02 & 0.02 & 0.03 & -0.03 & -0.07 & 1 & 0.16 \\
\hline 14) Race & 0.24 & $0.38^{*}$ & 0.12 & 0.12 & 0.10 & 0.04 & 0.08 & 0.11 & 0.11 & 0.13 & 0.13 & 0.06 & 0.16 & 1 \\
\hline
\end{tabular}

Note. ${ }^{\star}$ Significance attained at the $p<0.001$ level (2-tailed). Bold text indicates $p<0.01$. Italic font indicates $p<0.05$. Constructive and Unconstructive refer to the subscales of the Constructive and Unconstructive Worry Questionnaire. Safety and Avoidance Scales refer to the subscales of the Worry Behaviors Inventory. PSWQ = Penn State Worry Questionnaire. GAS = Geriatric Anxiety Scale. BMWS = Brief Measure of Worry Severity. WDQ-SF = Worry Domains Questionnaire Short Form. All results are pooled. Race was recoded as a binary variable (White and non-White) for correlational results due to small group sizes. 
To examine the relationship between worry constructiveness and overall anxiety, Pearson $r$ correlations were performed on GAS total scores with subdomain scores on the CUWQ. Among older adults, overall anxiety was significantly and positively associated with greater constructive worry $(r=0.24, p<0.05)$ with a small effect size, and with greater unconstructive worry $(r=0.78, p<$ 0.001 ) with a large effect size. Results were similar among younger adults, with overall anxiety being significantly and positively associated with greater constructive worry $(r=0.18, p<0.01)$ with a small effect size and greater unconstructive worry $(r=0.72, p<0.001)$ with a large effect size.

Finally, to investigate the relationships between responses to worry with overall severity of anxiety, Pearson $r$ correlations were performed on the subdomains of the WBI and GAS total scores among both younger and older adults. Among older adults, overall anxiety was significantly positively correlated with both safety behaviors $(r=0.57, p<0.001)$ and avoidance behaviors $(r=0.59, p<$ $0.001)$ with large effect sizes. Among younger adults, overall anxiety was similarly positively associated with both safety behaviors $(r=0.57, p<0.001)$ and avoidance behaviors $(r=0.50, p<0.001)$ with large effect sizes. Thus, the second hypothesis was also partially supported in that all measures of worry severity, unconstructive worry, and avoidance behaviors were significantly positively correlated with GAS total scores. However, constructive worry and safety behaviors were also unexpectedly positively correlated with GAS total scores.

\section{Discussion}

As anxiety is one of the most common mental health problems identified in older adults and worry is a cardinal symptom of anxiety disorders, the limited research examining worry among older adults represents a problematic gap. This study provided an in-depth analysis of worry as both its own construct characterized by a variety of facets as well as a symptom of anxiety to tease apart the multifaceted nature of worry.

\subsection{Age Differences on Worry Domains and Anxiety}

Consistent with the literature demonstrating that worry tends to decrease with age and is relatively infrequent in older adults (Goncalves \& Byrne, 2013; Golden et al., 2011), we found that younger adults consistently reported greater worry severity (as measured by the PSWQ, BMWS, and WDQ-SF) than older adults. Notably, however, the differences on select measures were less apparent among men than women, possibly due to men's tendency to report low worry in general, regardless of age. Younger adults also reported greater anxiety symptoms on the GAS, supporting previous research indicating that anxiety and other clinical symptoms tend to be greater in younger adults than older adults (Segal, Hook, \& Coolidge, 2001). Proposed explanations for these age differences include increased learned coping skills as individuals age (as discussed by Borkovec, 1988), differing presentations of worry and anxiety in older adults, decreased responsi- 
bilities in late life, and cohort effects between the current generations of older and younger adults (Basevitz, 2003).

Supporting these findings, we also found differences in worry content as younger adults consistently reported greater worry in all worry content areas (i.e., work, finances, aimless future, low confidence, relationships/social concerns) except health, where no significant difference was found between age groups. Notably, however, differences between age groups varied when gender was accounted for, as younger women tended to report the greatest worry on all domains sans health whereas both younger and older men consistently reported low worry across domains. Thus, age differences were most pronounced among women, who typically reported greater worry symptoms than men regardless of age. That being said, the most prominent areas of concern for each age group were consistent with much of the literature in that older adults reported the most worry about health whereas younger adults were most concerned about finances and social concerns, as is typically expected at these different stages of life (Hunt et al., 2009; Lindesay et al., 2006).

In addition to worry severity and content, we also examined two novel aspects of worry that have yet to garner much attention: constructiveness and response behaviors. Notably, age differences were demonstrated among unconstructive worry but not constructive worry. Younger adults reported greater unconstructive worry, consistent with findings of overall worry, however constructive worry was constant across age groups. These findings indicate that constructive worry may be a more universal construct and more akin to typical cognitive patterns across the lifespan compared to unconstructive worry which may better characterize the "worry" represented by most worry measures and definitions. Thus, these differences in unconstructive worry may be explained using the same hypotheses proposed in discussions of overall worry severity (e.g., cohort differences, accrued coping skills, decreased responsibilities, etc.). However, it should be noted that based on the current sample, it also seems that both younger and older adults commonly experience both constructive and unconstructive worry rather than one or the other independently. Therefore, further investigation may be warranted to understand the true nature of worry and its variants.

Another novel finding of the current study is that younger adults report using both greater safety and avoidance behaviors than older adults on the WBI, contrary to our hypothesis that older adults would utilize greater safety behaviors due to learned coping across the lifespan. However, as younger adults also reported experiencing greater worry (especially unconstructive worry) and anxiety, it may be that they have greater need for strategies to manage their symptoms and may thus demonstrate a wider variety of responses to worry as opposed to older adults who worry infrequently and may not need to use these strategies regularly. It should also be noted that when accounting for gender, this difference was only found among women in our sample, with no differences found 
between younger and older men's use of response behaviors. Thus, further exploration may be warranted to understand gender's role in determining worry response patterns.

\subsection{Relationships between Anxiety and Worry Domains}

As expected, worry severity was consistently significantly and positively associated with overall anxiety across a variety of measures in both age groups in the current study. As worry represents a cardinal symptom of anxiety disorders, these results support the previous literature and are consistent with the current understanding of the psychopathology of anxiety across age groups. To take this a step further, however, some interesting relationships between anxiety and specific aspects of worry were also found in the current study. For example, overall anxiety was significantly positively correlated with unconstructive worry in both younger and older adults as predicted but was also unexpectedly positively correlated with constructive worry among both samples as well. Thus, both forms of worry may be related to overall worry and anxiety, rather than solely unconstructive thought patterns. Therefore, it is important to understand how adaptive worry patterns may also drive measurement of general worry and anxiety and may even offer ways to incorporate positive cognitive strategies that individuals are already utilizing into intervention. Alternatively, these findings may imply that the distinction between constructive and unconstructive worry is not clear-cut and may be difficult to measure independently, as both were related to anxiety in general, though at different levels. Supporting this assertion, simple correlations indicated that constructive and unconstructive worry were significantly and positively correlated among both samples in the present study, consistent with findings from one of the two original studies examining the CUWQ (McNeill \& Dunlop, 2016). Thus, as indicated by the original authors, the relationship between constructive and unconstructive worry seems to be more complex than simply being opposites of one another.

Finally, an interesting relationship between overall anxiety and worry response behaviors was found among both younger and older adults. Anxiety was significantly positively associated with both avoidance and safety behaviors, contrary to the hypothesis that safety behaviors function to mitigate anxiety symptoms. This provides support for the idea that safety behaviors in particular may be ubiquitous strategies used by most individuals, regardless of anxiety symptoms. This finding also reinforces the idea that individuals with more worry and anxiety engage in greater worry management behaviors in general due to the higher needs placed on them by greater anxious distress. This hypothesis is consistent with previous research that indicates that in adolescents, those experiencing substantial anxiety report engaging in significantly more coping strategies, both positive (i.e., planning, acceptance, positive refocusing) and negative (i.e., rumination, self-blame, catastrophizing), than those without anxiety (Legerstee et al., 2011). 


\section{Practical Implications}

Several practical implications of the current findings should be noted. First, it is suggested that in the presence of anxiety, worry and most notably worry content, severity, and constructiveness, should also be assessed. Based on our findings, specific characteristics of worry (e.g., unconstructive worry, significant worry about health, heightened use of response behaviors) appear to be associated with elevated anxiety among older adults and thus researchers and clinicians may benefit from assessing for these indicators. Though the current study did not examine clinical samples, one might infer that by thoroughly assessing diverse aspects of worry, mental health professionals may benefit from more comprehensive case formulations and targeted treatment planning and interventions for older adults experiencing anxiety symptoms. Another implication of the current study is the necessity of considering the interaction of gender and age on the experience of worry and anxiety. Gender was indicated to play a major role in the presentation of worry and anxiety across age groups. Thus, anxiety should be viewed with the broader life context of the individual in mind, considering gender and age as interacting influences rather than independent factors in predicting worry and anxiety expression.

\section{Limitations}

Strengths of the current study include the inclusion of diverse measures of worry and the relatively large sample size. In addition, the current study provides evidence of reliability and validity for use of the primary study measures with older adult populations, as several measures appear to have never been specifically examined among older adults. However, several limitations should also be noted. Participants in the current study were primarily White, highly educated, working- and middle-class individuals. Thus, inherent characteristics of the samples in this study (e.g., race/ethnicity, education level, SES) may limit the generalizability of our findings. Further studies should seek to expand the current study with more diverse populations including greater racial/ethnic diversity, a broader range of education levels and socioeconomic statuses, and the in clusion of additional samples of individuals with clinical levels of anxiety symptoms to assess a broader spectrum of anxiety. Additionally, demographic differences between the younger and older samples used in the study represent a significant limitation. For example, although race was not found to be significantly associated with the outcome variables and gender was statistically accounted for within cross-sectional analyses, the greater racial diversity and larger proportion of female respondents in the younger adult sample constitute sample disparities that may influence the comparability of our samples. The current study also used only self-report measures of worry and anxiety. Future studies might seek to include additional methods of measuring these constructs such as keeping worry logs, examining immediate worry/anxiety responses and behaviors via experimental methods, or through clinical interviews. Finally, due to the cross-sectional 
design in the current study, causal inferences among the variables cannot be determined. Thus, future studies should further tease apart the causality and directionality involved in older adults' experiences of anxiety and worry in prospective and longitudinal designs. These limitations considered, the current study serves as an initial examination of the multifaceted aspects of worry, and we believe that further empirical scrutiny of worry as a multidimensional construct is needed.

\section{Conflicts of Interest}

The authors declare no conflicts of interest regarding the publication of this paper.

\section{References}

Basevitz, P. (2003). Worry in Late Adulthood: Developmental Perspectives on Content, Frequency, and Worry Proneness. Doctoral Dissertation, Montreal: Concordia University.

Borkovec, T. D. (1988). Comments on "Worry as a Phenomenon Relevant to the Elderly". Behavior Therapy, 19, 381-383. https://doi.org/10.1016/S0005-7894(88)80010-8

Borkovec, T. D., Alcaine, O., \& Behar, E. S. (2004). Avoidance Theory of Worry and Generalized Anxiety Disorder. In R. Heimberg, D. Mennin, \& C. Turk (Eds.), Generalized Anxiety Disorder: Advances in Research and Practice (pp. 77-108). New York: Guilford.

Borkovec, T. D., Robinson, E., Pruzinsky, T., \& DePree, J. A. (1983). Preliminary Exploration of Worry: Some Characteristics and Processes. Behaviour Research and Therapy, 21, 9-16. https://doi.org/10.1016/0005-7967(83)90121-3

Borkovec, T. D., Shadick, R. N., \& Hopkins, M. (1991). The Nature of Normal and Pathological Worry. In R. M. Rapee, \& D. H. Barlow (Eds.), Chronic Anxiety: Generalized Anxiety Disorder and Mixed Anxiety-Depression (pp. 29-51). New York: Guilford Press.

de Vito, A., Calamia, M., Greening, S., \& Roye, S. (2017). The Association of Anxiety, Depression, and Worry Symptoms on Cognitive Performance in Older Adults. Aging, Neuropsychology, and Cognition, 26, 161-173. https://doi.org/10.1080/13825585.2017.1416057

Diefenbach, G. J., McCarthy-Larzelere, M. E., Williamson, D. A., Mathews, A., Manguno-Mire, G. M., \& Bentz, B. G. (2001). Anxiety, Depression, and the Content of Worries. Depression and Anxiety, 14, 247-250. https://doi.org/10.1002/da.1075

Dugas, M. J., Gagnon, F., Ladouceur, R., \& Freeston, M. H. (1998). Generalized Anxiety Disorder: A Preliminary Test of a Conceptual Model. Behaviour Research and Therapy, 36, 215-226. https://doi.org/10.1016/S0005-7967(97)00070-3

Fox, E., Dutton, K., Yates, A., Georgiou, G. A., \& Mouchlianitis, E. (2015). Attentional Control and Suppressing Negative Thought Intrusions in Pathological Worry. Clinical Psychological Science, 3, 593-606. https://doi.org/10.1177/2167702615575878

Gladstone, G. L., Parker, G. B., Mitchell, P. B., Malhi, G. S., Wilhelm, K. A., \& Austin, M. P. (2005). A Brief Measure of Worry Severity (BMWS): Personality and Clinical Correlates of Severe Worriers. Journal of Anxiety Disorders, 19, 877-892. https://doi.org/10.1016/j.janxdis.2004.11.003

Golden, J., Conroy, R. M., Bruce, I., Denihan, A., Greene, E., Kirby, M., \& Lawlor, B. A. 
(2011). The Spectrum of Worry in the Community-Dwelling Elderly. Aging \& Mental Health, 15, 985-994. https://doi.org/10.1080/13607863.2011.583621

Goncalves, D. C., \& Byrne, G. J. (2013). Who Worries Most? Worry Prevalence and Patterns across the Lifespan. International Journal of Geriatric Psychiatry, 28, 41-49. https://doi.org/10.1002/gps.3788

Gould, C. E., Gerolimatos, L. A., Beaudreau, S. A., Mashal, N., \& Edelstein, B. A. (2018). Older Adults Report More Sadness and Less Jealousy than Young Adults in Response to Worry Induction. Aging \& Mental Health, 22, 512-518. https://doi.org/10.1080/13607863.2016.1277975

Hunt, S. L., Wisocki, P. A., \& Roger, P. R. (2009). “What, Me Worry?” An Examination of the Literature on Worry and the Older Adult. Behavior Therapist, 32, 9-16.

Jeon, H. S., Dunkle, R., \& Roberts, B. L. (2006). Worries of the Oldest-Old. Health \& Social Work, 31, 256-265. https://doi.org/10.1093/hsw/31.4.256

Legerstee, J. S., Garnefski, N., Verhulst, F. C., \& Utens, E. M. W. J. (2011). Cognitive Coping in Anxiety-Disordered Adolescents. Journal of Adolescence, 34, 319-326. https://doi.org/10.1016/j.adolescence.2010.04.008

Lindesay, J., Baillon, S., Brugha, T., Dennis, M., Stewart, R., Araya, R., \& Meltzer, H. (2006). Worry Content across the Lifespan: An Analysis of 16- to 74-Year-Old Participants in the British National Survey of Psychiatric Morbidity 2000. Psychological Medicine, 36, 1625-1633. https://doi.org/10.1017/S0033291706008439

Mahoney, A. E., Hobbs, M. J., Newby, J. M., Williams, A. D., \& Andrews, G. (2018). Psychometric Properties of the Worry Behaviors Inventory: Replication and Extension in a Large Clinical and Community Sample. Behavioural and Cognitive Psychotherapy, 46, 84-100. https://doi.org/10.1017/S1352465817000455

Mahoney, A. E., Hobbs, M. J., Newby, J. M., Williams, A. D., Sunderland, M., \& Andrews, G. (2016). The Worry Behaviors Inventory: Assessing the Behavioral Avoidance Associated with Generalized Anxiety Disorder. Journal of Affective Disorders, 203, 256-264. https://doi.org/10.1016/j.jad.2016.06.020

McNeill, I. M., \& Dunlop, P. D. (2016). Development and Preliminary Validation of the Constructive and Unconstructive Worry Questionnaire: A Measure of Individual Differences in Constructive versus Unconstructive Worry. Psychological Assessment, 28, 1368-1378. https://doi.org/10.1037/pas0000266

Mennin, D. S., Heimberg, R. G., Turk, C. L., \& Fresco, D. M. (2005). Preliminary Evidence for an Emotion Dysregulation Model of Generalized Anxiety Disorder. Behaviour Research and Therapy, 43, 1281-1310. https://doi.org/10.1016/j.brat.2004.08.008

Meyer, T. J., Miller, M. L., Metzger, R. L., \& Borkovec, T. D. (1990). Development and Validation of the Penn State Worry Questionnaire. Behaviour Research and Therapy, 28, 487-495. https://doi.org/10.1016/0005-7967(90)90135-6

Miloyan, B., \& Pachana, N. A. (2016). Clinical Significance of Individual GAD Symptoms in Later Life. Journal of Geriatric Psychiatry and Neurology, 29, 92-98. https://doi.org/10.1177/0891988715606231

Newman, M. G., \& Llera, S. J. (2011). A Novel Theory of Experiential Avoidance in Generalized Anxiety Disorder: A Review and Synthesis of Research Supporting a Contrast Avoidance Model of Worry. Clinical Psychology Review, 31, 371-382. https://doi.org/10.1016/j.cpr.2011.01.008

Nilsson, J., Sigström, R., Östling, S., Waern, M., \& Skoog, I. (2019). Changes in the Expression of Worries, Anxiety, and Generalized Anxiety Disorder with Increasing Age: A Population Study of 70 to 85-Year-Olds. International Journal of Geriatric Psychiatry, 34, 249-257. https://doi.org/10.1002/gps.5012 
Ottaviani, C., Borlimi, R., Brighetti, G., Caselli, G., Favaretto, E., Giardini, I., Marzocchi, C., Nucifora, V., Rebecchi, D., Ruggiero, G. M., \& Sassaroli, S. (2014). Worry as an Adaptive Avoidance Strategy in Healthy Controls but Not in Pathological Worriers. International Journal of Psychophysiology, 93, 349-355.

https://doi.org/10.1016/j.ijpsycho.2014.05.010

Pietrzak, R. H., Maruff, P., Woodward, M., Fredrickson, J., Fredrickson, A., Krystal, J. H., Southwick, S. M., \& Darby, D. (2012). Mild Worry Symptoms Predict Decline in Learning and Memory in Healthy Older Adults: A 2-Year Prospective Cohort Study. The American Journal of Geriatric Psychiatry, 20, 266-275.

https://doi.org/10.1097/JGP.0b013e3182107e24

Ruscio, A. M. (2002). Delimiting the Boundaries of Generalized Anxiety Disorder: Differentiating High Worriers with and without GAD. Journal of Anxiety Disorders, 16, 377-400. https://doi.org/10.1016/S0887-6185(02)00130-5

Segal, D. L., Granier, K. L., Stone, L. E., \& Pifer, M. (2019). Geriatric Anxiety Scale. In D. $\mathrm{Gu}, \&$ M. Dupree (Eds.), Encyclopedia of Gerontology and Population Aging. Basingstoke: Springer Nature. https://doi.org/10.1007/978-3-319-69892-2_1103-1

Segal, D. L., Hook, J. N., \& Coolidge, F. L. (2001). Personality Dysfunction, Coping Styles, and Clinical Symptoms in Younger and Older Adults. Journal of Clinical Geropsychology, 7, 201-212. https://doi.org/10.1023/A:1011391128354

Segal, D. L., June, A., Payne, M., Coolidge, F. L., \& Yochim, B. (2010). Development and Initial Validation of a Self-Report Assessment Tool for Anxiety among Older Adults: The Geriatric Anxiety Scale. Journal of Anxiety Disorders, 24, 709-714.

https://doi.org/10.1016/j.janxdis.2010.05.002

Spinhoven, P., van der Veen, D. C., Voshaar, R. O., \& Comijs, H. C. (2017). Worry and Cognitive Control Predict Course Trajectories of Anxiety in Older Adults with Late-Life Depression. European Psychiatry, 44, 134-140.

https://doi.org/10.1016/j.eurpsy.2017.05.002

Stöber, J., \& Joormann, J. (2001). A Short Form of the Worry Domains Questionnaire: Construction and Factorial Validation. Personality and Individual Differences, 31, 591-598. https://doi.org/10.1016/S0191-8869(00)00163-X

Tallis, F., Eysenck, M., \& Mathews, A. (1992). A Questionnaire for the Measurement of Nonpathological Worry. Personality and Individual Differences, 13, 161-168.

https://doi.org/10.1016/0191-8869(92)90038-Q

Tully, P. J., Cosh, S. M., \& Baune, B. T. (2013). A Review of the Effects of Worry and Generalized Anxiety Disorder upon Cardiovascular Health and Coronary Heart Disease. Psychology, Health \& Medicine, 18, 627-644. https://doi.org/10.1080/13548506.2012.749355

Wisocki, P. A. (1994). The Experience of Worry among the Elderly. In G. C. L. Davey, \& F. Tallis (Eds.), Wiley Series in Clinical Psychology. Worrying: Perspectives on Theory, Assessment and Treatment (pp. 247-261). Oxford: John Wiley \& Sons.

Wisocki, P. A., Handen, B., \& Morse, C. K. (1986). The Worry Scale as a Measure of Anxiety among Homebound and Community Active Elderly. The Behavior Therapist, 9, 91-95.

Wu, M., Mennin, D. S., Ly, M., Karim, H. T., Banihashemi, L., Tudorascu, D. L., Aizenstein, H. J., \& Andreescu, C. (2019). When Worry May Be Good for You: Worry Severity and Limbic-Prefrontal Functional Connectivity in Late-Life Generalized Anxiety Disorder. Journal of Affective Disorders, 257, 650-657.

https://doi.org/10.1016/j.jad.2019.07.022 